\title{
Antitumour and antiangiogenic effects of IDN 5390, a novel C-seco taxane, in a paclitaxel-resistant human ovarian tumour xenograft
}

\author{
G Petrangolini', G Cassinelli', G Pratesi*,', M Tortoreto', E Favini', R Supino', C Lanzi', S Belluco² and \\ F Zunino'
}

'Department of Experimental Oncology, Istituto Nazionale Tumori, via Venezian I, 20133 Milan, Italy; ${ }^{2}$ Department of Veterinary Pathology, Hygiene and Public Health, University of Milan, 20133 Milan, Italy

IDN 5390 is a novel C-seco taxane analogue selected for preclinical development on the basis of its antimotility activity on endothelial cells, antitumour efficacy in a large panel of human tumour xenografts and high tolerability in mouse. On the basis of oral availability, IDN 5390 is suitable for protracted administration schedules. Such a treatment schedule has been reported as the most appropriate to exploit the antiangiogenic effects of cytotoxic drugs. An ability to downregulate angiogenesis-related growth factors in tumour cells has been described for IDN 5390. The aim of the study was to investigate the antitumour and antiangiogenic potential of oral IDN 5390 on a human ovarian carcinoma xenograft, the INT.ACP/PTX, resistant to paclitaxel (PTX). Such tumour line was derived in vivo from a cisplatin-resistant tumour line, the A2780/DDP, which is sensitive to PTX. Compared to the parental cells, INT.ACP/PTX cells exhibited a high level of Pgp expression, resulting in a reduced in vitro sensitivity to both PTX and IDN 5390. The INT.ACP/PTX tumour xenograft was still resistant to PTX, but responsive to IDN 5390, when delivered per os, by a daily prolonged schedule. A direct effect on tumour cells, allowed by the high tolerability of the compound in mouse, cannot be excluded in vivo. Immunohistochemical analysis indicated a significant reduction of microvessel density in IDN 5390-treated tumours, lasting till 7 days after the last drug administration. Thus, a prolonged inhibitory effect on tumour angiogenesis is consistent with the persistent growth control of INT.ACP/PTX tumour achieved by IDN 5390. On the contrary, the low tolerability and the limited oral availability of conventional taxanes do not allow an easy feasibility of such treatment regimen. Thus, the tolerability profile of IDN 5390 in preclinical systems and its efficacy in PTX-resistant tumours support the therapeutic interest for its clinical development, with particular attention to oral daily prolonged schedules.

British Journal of Cancer (2004) 90, |464-|468. doi:I0.1038/sj.bjc.660 I730 www.bjcancer.com

Published online 9 March 2004

(c) 2004 Cancer Research UK

Keywords: taxanes; IDN 5390; angiogenesis; MDR; in vivo systems

Taxanes are effective antitumour agents characterised by a unique mechanism of action, that is, microtubule polymerisation resulting in cell death following arrest of cell cycle in $\mathrm{M}$ phase and formation of aberrant mitoses (Horwitz et al, 1993; Jordan et al, 1993). In addition to the cytotoxic effects on tumour cells, effects on angiogenesis have been reported for paclitaxel (PTX) and docetaxel, the two taxanes currently used in clinics (Verweij et al, 1994; Rowinsky, 1997). These effects have been related to the ability of inhibiting endothelial cell motility (Belotti et al, 1996; Sweeney et al, 2001) and downregulating angiogenesis-related growth factors (Cassinelli et al, 2002). The novel C-seco taxane IDN 5390, which is currently under study in preclinical systems, was originally selected for its potent antimotility activity and low cytotoxicity in endothelial cells (Taraboletti et al, 2002). In vivo studies in human tumour xenografts have indicated a unique profile of tolerability and antitumour activity for IDN 5390 when

*Correspondence: G Pratesi; E-mail: graziella.pratesi@istitutotumori.mi.it Received 17 October 2003; revised 29 December 2003; accepted 28 January 2004; published online 9 March 2004 administered by a daily schedule for a prolonged time (Pratesi et al, 2003).

In recent years, many preclinical studies have indicated the possibility of using cytotoxic drugs as antiangiogenic agents for the antitumour therapy. Chronic and frequent treatment schedules have been reported as the most appropriate to exploit the antiangiogenic effect of cytotoxic drugs, compared to the highdose/intermittent-schedule mainly targeting tumour cells (Hanahan et al, 2000; Miller et al, 2001). Conventional antitumour drugs delivered according to the so-called 'metronomic chemotherapy' were effective even against drug-resistant tumours (Browder et al, 2000; Fidler and Ellis, 2000). When administered according to a frequent and prolonged schedule, IDN 5390 is active against all human tumour xenografts investigated, including the PTXresistant ones (Pratesi et al, 2003).

The aim of the study was to further investigate the mechanisms responsible for the antitumour activity of IDN 5390 in PTXresistant human tumour xenografts and, in particular, in the ovarian carcinoma INT.ACP/PTX. Such tumour line was derived in vivo by one single s.c. growing A2780/DDP tumour xenograft (cisplatin-resistant) after treatment with PTX. The mechanisms of 
resistance of these tumour cells and the in vivo antiangiogenic effect of the drug were investigated. The results are consistent with a contribution of antiangiogenic effects in the antitumour efficacy of the taxane analogue.

\section{MATERIALS AND METHODS}

\section{Drugs, tumour and cells}

IDN 5390, (13-( $N$-Boc- $\beta$-isobutylisoserinyl)-10 $\beta$-dehydro-C-seco10-deacetylbaccatin III) and PTX, used as reference drug, were provided by Indena S. p.a. (Milan, Italy) (Figure 1). For in vitro studies, drugs were dissolved in dimethylsulphoxide (DMSO) at $2 \mathrm{mg} \mathrm{ml}^{-1}$ and diluted in culture medium (DMSO final concentration $0.25 \%$ ). For in vivo studies, PTX was dissolved by adding absolute ethanol and Cremophor ELP (both 5\% of the final volume) (Polizzi et al, 1999), whereas IDN 5390, dissolved in Polysorbate 80 , was diluted just before use by adding $0.9 \% \mathrm{NaCl}$ to the final concentration of $10 \% \mathrm{vv}^{-1}$ (Pratesi et al, 2003).

The study was performed on the A2780 human ovarian carcinoma cell line and two variants, that is, the A2780/DDP cell subline, selected in vitro for resistance to cisplatin (Beherens et al, 1987), and the INT.ACP/PTX cell subline. The latter variant was derived from a single A2780/DDP tumour xenograft which, unexpectedly, was not responding to PTX treatment in vivo. Indeed, the A2780/DDP tumour line is highly sensitive to PTX treatment (Polizzi et al, 1999). The line was maintained either by s.c. passages of tumour fragments (tumour line) or as cell culture (cell line). A2780/DX, selected in vitro for acquired resistance to doxorubicin, was used as positive control for the Pgp expression analysis.

All cell lines were maintained in RPMI 1640 (Bio-Whittaker Verviers, Belgium) supplemented with $10 \%$ foetal calf serum (Life Technologies, Gaithersburg, MD, USA) in $5 \% \mathrm{CO}_{2}$ atmosphere.

\section{In vitro studies}

Tumour cell sensitivity to IDN 5390 and PTX was evaluated by cell growth inhibition assay. Cells were seeded in six-well plates in duplicate and after $24 \mathrm{~h}$ exposed to the solvent or to the drugs at different concentrations. After $72 \mathrm{~h}$, cells were trypsinised and counted by a Coulter Counter (Coulter Electronics, Luton, UK). The concentration able to inhibit cell proliferation by $50 \%\left(\mathrm{IC}_{50}\right)$ was derived by dose/effect plots. Resistant Index (RI) was assessed as the ratio between the drug $\mathrm{IC}_{50}$ in each cell subline and in the parental A2780 cell line.

For the MDR phenotype characterisation, the Pgp expression was determined by immunofluorescence (Gottesman and Pastan, 1993). Cells $\left(10^{6}\right)$ were trypsinised and washed in PBA (phosphatebuffered saline (PBS) and 1\% bovine serum albumin (BSA)). Cells were then incubated in $100 \mu \mathrm{l}$ of PBA containing $10 \mu \mathrm{l}$ of gp170FITC antibody (YLEM, Rome, Italy). After washing in PBS, expression of the protein was assessed as fluorescence intensity using a FACScan (Becton Dickinson, Mountain View, CA, USA).
Paclitaxel

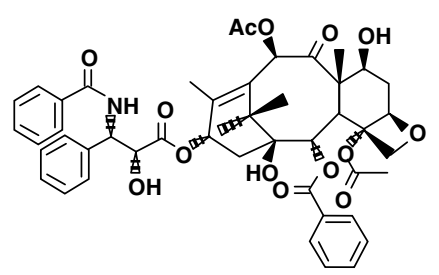

IDN 5390

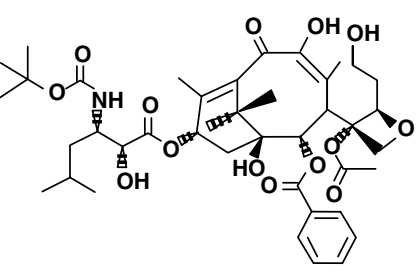

Figure I Chemical structures of paclitaxel and IDN 5390.
Bcl-2 expression was examined in whole-cell extracts prepared as previously reported in Pratesi et al (2003). Briefly, equal amounts of proteins were separated by SDS-PAGE and transferred onto nitrocellulose sheets. Then filters were incubated with mouse monoclonal anti-Bcl-2 antibody (Santa Cruz Biotechnology, CA, USA) or with rabbit polyclonal antiactin (Sigma, St Louis, MO, USA). Immunocomplexes were visualised by the Pierce Super Signal System (Pierce, Rockford, IL, USA).

\section{In vivo studies}

All the experiments were carried out using adult ( $8-10$ weeks old) female athymic CD-1 nude mice (Charles River, Calco, Italy). Mice were maintained in laminar flow rooms at constant temperature and humidity, with free access to sterilised food and water. Experimental protocols were approved by the Ethic Committee for Animal Experimentation of our Institute (Istituto Nazionale per lo Studio e la Cura dei Tumori, Milan, Italy), according to the United Kingdom Coordinating Committee on Cancer Research Guidelines (Workman et al, 1998).

The INT.ACP/PTX tumour was maintained in line by subsequent fragment implants, as previously described (Pratesi et al, 1989). At each passage mice were treated with PTX (i.v., $36 \mathrm{mg} \mathrm{kg}^{-1}, 7$ days after tumour inoculum), and in such conditions tumour weight inhibition (TWI) induced by the optimal PTX regimen was lower than $50 v s$ a $94 \%$ inhibition in the A2780/DDP tumour line (Polizzi et al, 1999).

Drug solutions of 3.6 and $9 \mathrm{mg} \mathrm{ml}^{-1}$ were prepared, as described above, for PTX and IDN 5390, respectively, corresponding to 36 and $90 \mathrm{mg} \mathrm{kg}^{-1}$ for an administration volume of $10 \mathrm{ml} \mathrm{kg}^{-1}$ of body weight. Larger volumes were injected for higher doses.

For experimental purposes, tumour fragments were s.c. implanted in the right flank of mice. Tumour growth was followed by biweekly measurements of tumour diameters with a Vernier caliper. Tumour weight (TW) was calculated according to the formula: TW $(\mathrm{mg})=$ tumour volume $\left(\mathrm{mm}^{3}\right)=d^{2} \times D / 2$, where $d$ and $D$ are the shortest and the longest diameter, respectively. Mice bearing tumours of $250-300 \mathrm{mg}$ were treated with IDN 5390 and PTX, according to different treatment routes (i.v., s.c. or p.o.) and schedules (daily, i.e. $\mathrm{qd} \times 8$, or intermittent, i.e. $\mathrm{q} 4 \mathrm{~d} \times 3$ ). The first and the last day of treatment were the same for the two schedules. At 1 and 7 days after treatment end, three mice/group were killed by cervical dislocation. Tumours were excised, weighed, fixed in zinc fixative and stained with standard haematoxylin-eosin in order to study the overall tissue morphology. Angiogenesis was assessed by immunohistochemistry (IHC), staining blood vessels with a rat anti-mouse CD31/PECAM-1 monoclonal antibody (kindly supplied by Dr A Vecchi, Mario Negri Institute, Milan, Italy), as previously described (Cassinelli et al, 2002). Microvessel density (MVD) was determined using a WebSlide Browser software. Briefly, microvessels were quantified within six random fields $\left(0.159 \mathrm{~mm}^{2}\right.$ fields, $\times 200$ magnification $)$ selected for high vascularisation (hot spot areas). Microvessel density was expressed as mean number \pm s.d. Percentage MV inhibition (MVI\%) in drug-treated vs control mice was calculated as MVI\% $=100-($ mean MVD in treated/mean MVD in control tumours $\times 100$ ). Neither vessel lumen nor red blood cells were used to define a microvessel. Scoring of histological tumour sections was performed by two independent observers, without knowledge of the experimental group, with an interobserver reproducibility $>95 \%$.

Drug efficacy was assessed, at the last day of treatment, as percentage TW inhibition (TWI\%) expressed as TWI\% $=100-$ (mean TW treated/mean TW control $\times 100$ ).

For statistical analysis, TW and MV number were compared in treated $v s$ control mice by the unpaired Student's $t$-test (twotailed). 


\section{RESULTS}

\section{Chemosensitivity and drug resistance}

Pgp overexpression is a frequent mechanism of the cellular resistance to hydrophobic drugs, including PTX (Horwitz et al, 1993; Zunino et al, 1999). Thus, the Pgp expression levels were investigated by FACS analysis in the parental A2780 and in its variant cell lines (Figure 2). Pgp was expressed at low level in A2780 and A2780/DDP cells (mean fluorescence intensity being 8.5 and 7.9, respectively), whereas an increased expression was found in INT.ACP/PTX cells (mean fluorescence intensity, 25). A2780/DX cells were used as a positive control because of their MDR phenotype (mean fluorescence intensity, 140). Since Pgp expression can be modulated by treatment with drugs that are substrates for the transport system (Gottesman and Pastan, 1993), the protein level was examined after $72 \mathrm{~h}$-treatment with PTX or IDN 5390. As shown in Figure 3 an increased Pgp level was found in A2780/DDP cells after exposure to taxanes, whereas no significant modulation was observed in A2780 (not shown) and INT.ACP/PTX cells.

The antiproliferative effects of PTX and IDN 5390 after $72 \mathrm{~h}$ of treatment indicated an inverse correlation between Pgp expression and cell sensitivity to the taxanes (Table 1). Thus, A2780/DX and INT.ACP/PTX cells exhibited a reduced sensitivity to both taxanes. However, a lower Resistance Index (RI) was observed for IDN 5390 than for PTX. Co-treatment of INT.ACP/PTX cells with each taxane and the pump-antagonist verapamil $(10 \mu \mathrm{M})$ resulted in a complete reversal of resistance, with $\mathrm{IC}_{50}$ values similar to those in the A2780/DDP cell line, that is, $17.5 \pm 1.2$ and $57 \pm 4 \mathrm{nM}$ for PTX and IDN 5390, respectively (not shown).

A differential expression of $\mathrm{Bcl}-2$ protein was detected by Western Blot analysis in the cell lines. A2780/DDP cell lines showed increased basal levels of this protein compared to the parental A2780 cell line. No Bcl-2 protein was detectable in INT.ACP/PTX cells by this technique (Figure 4).

\section{In vivo studies}

We have already reported that the INT.ACP/PTX tumour xenograft is resistant to PTX (less than $50 \%$ TWI), but highly sensitive $(87 \%$ TWI) to IDN $5390,120 \mathrm{mg} \mathrm{kg}^{-1}$, delivered twice a day for 15 days (Pratesi et al, 2003). In the present study, the same dose of IDN 5390 was delivered only once a day for eight treatments, and a lower antitumour effect (60\% TWI) was achieved (Table 2). Anyway, IDN 5390 resulted much more effective than PTX delivered in similar conditions $(P<0.005)$, or at its best regimen $(P<0.05)$.

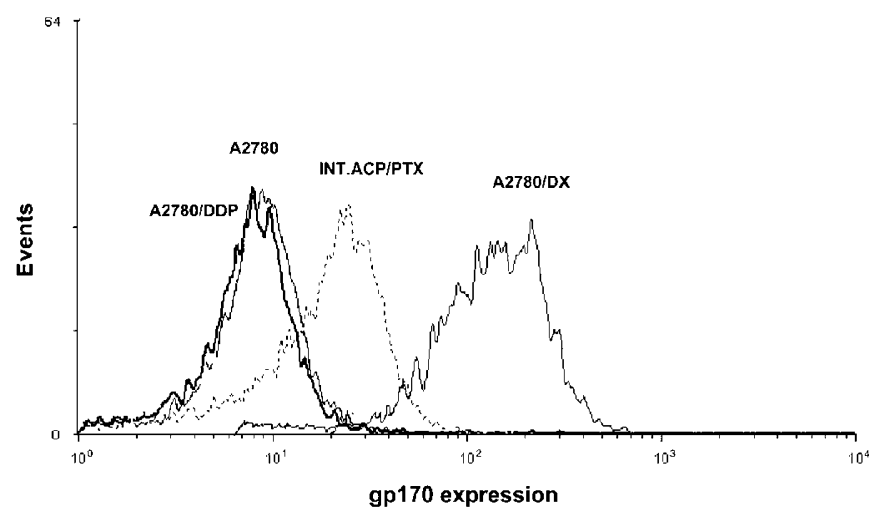

Figure 2 Pgp expression in the A2780 and its variant cell lines. A2780/ DX cells were used as positive control. Protein expression was assessed as fluorescence intensity in logarithmically growing cells stained with gp I70FITC antibody. One experiment representative of two for each cell line is reported.
A prolonged treatment schedule, which resulted to be the optimal one for the antitumour efficacy of IDN 5390, has been reported as the most suitable for exploiting the antiangiogenic effect of cytotoxic drugs. Thus, the effect on angiogenesis of the

\section{A2780/DDP}
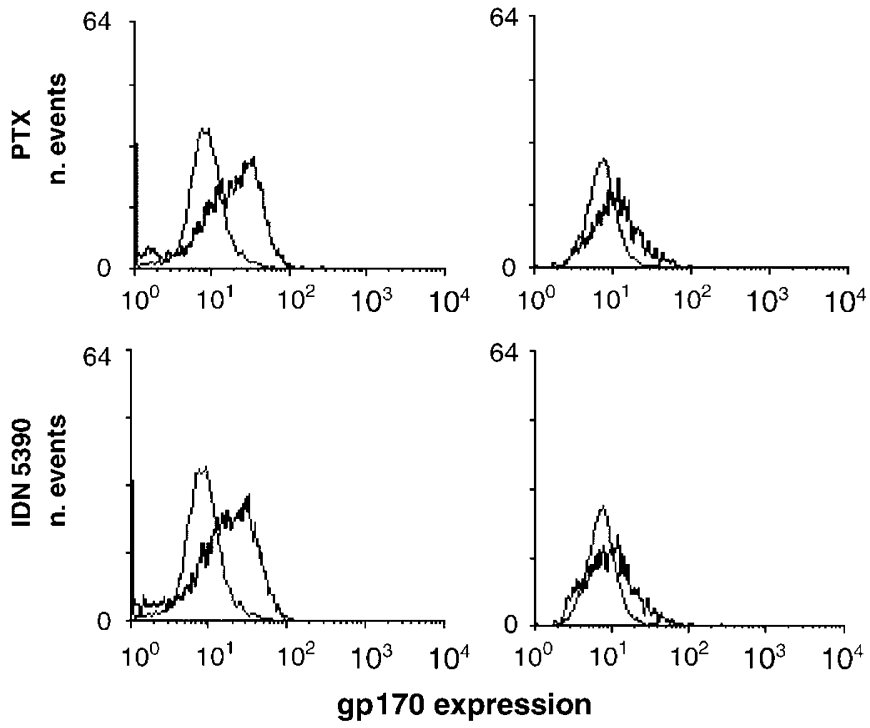

Figure 3 Pgp expression in A2780/DDP and INT.ACP/PTX cell lines treated with PTX or IDN 5390. Protein expression was assessed as fluorescence intensity in cells treated with the $I_{80}$ of each drug for $72 \mathrm{~h}$ and stained with gp I 70-FITC antibody. One experiment representative of two for each cell line is reported.

Table I Antiproliferative activity of IDN 5390 and PTX on different human ovarian tumour cell lines

\begin{tabular}{lccccc}
\hline & \multicolumn{2}{c}{$\mathbf{P T X}$} & & \multicolumn{2}{c}{ IDN $\mathbf{5 3 9 0}$} \\
\cline { 2 - 3 } & $\mathbf{I C}_{\mathbf{5 0}}^{\mathbf{a}} \mathbf{( n M )}$ & $\mathbf{R I}^{\mathbf{b}}$ & & $\mathbf{I C}_{\mathbf{5 0}}^{\mathbf{a}} \mathbf{( n M )}$ & $\mathbf{R I}^{\mathbf{b}}$ \\
\hline A2780 & $9.5 \pm 1$ & & & $29 \pm 0.5$ & \\
A2780/DDP & $13.8 \pm 1.7$ & 1.5 & & $40.4 \pm 7$ & 1.4 \\
INT.ACP/PTX & $140 \pm 23$ & 15 & & $203 \pm 35.5$ & 7 \\
A2780/DX & $366 \pm 30$ & 38.6 & & $424 \pm 44.4$ & 14.5 \\
\hline
\end{tabular}

${ }^{a} C_{50}$ : drug concentration required to inhibit $50 \%$ of cell growth compared to untreated cells $(72 \mathrm{~h}$ of drug exposure). Mean \pm s.d. of at least three independent experiments are reported. ${ }^{b}$ Resistance Index (RI), calculated as the ratio between the $I C_{50}$ of the cell sublines and the $I_{50}$ of the parental A2780 cell line.

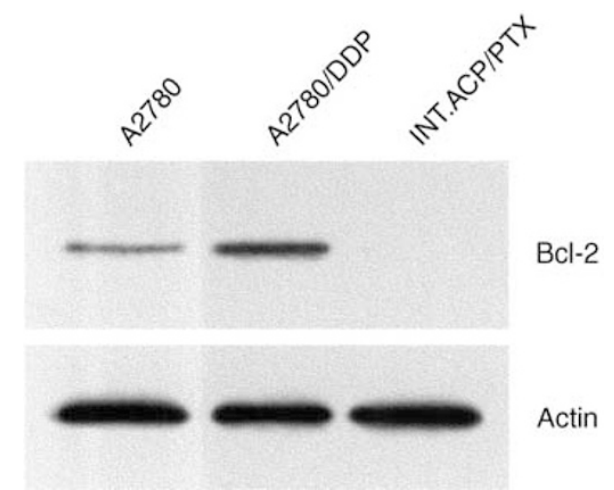

Figure 4 Expression of $\mathrm{BCl}-2$ protein in A2780 and its variant cell lines. Equal amounts of proteins were separated by SDS-PAGE and analysed by immunoblotting. Control for protein loading by actin is shown. 
Table 2 Antitumour and antiangiogenic effects of IDN 5390 and PTX delivered by different treatment schedules against the INT.ACP/PTX human ovarian tumour xenograft

\begin{tabular}{|c|c|c|c|c|c|c|c|c|}
\hline Drug & Route & \multicolumn{2}{|c|}{ Dose $\left(\mathrm{mg} \mathrm{kg}^{-1}\right)$} & \multicolumn{2}{|c|}{ Treatment } & TWI\% ${ }^{a}$ & \multicolumn{2}{|c|}{ MVI\% ${ }^{\mathbf{b}}$} \\
\hline IDN 5390 & p.o. & 120 & 960 & Daily × 8 & $4-11$ & 60 & $55^{*} *$ & $26 *$ \\
\hline
\end{tabular}

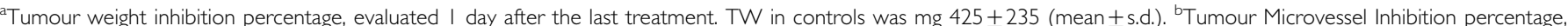

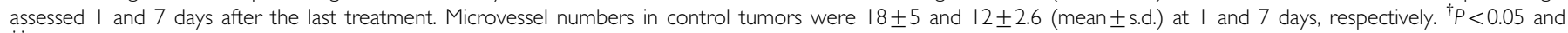
${ }^{\dagger \dagger} P<0.005$ vs IDN 5390 -treated mice; $* P<0.05$ and $* * P<0.000$ I vs control tumours, by Student's $t$-test.
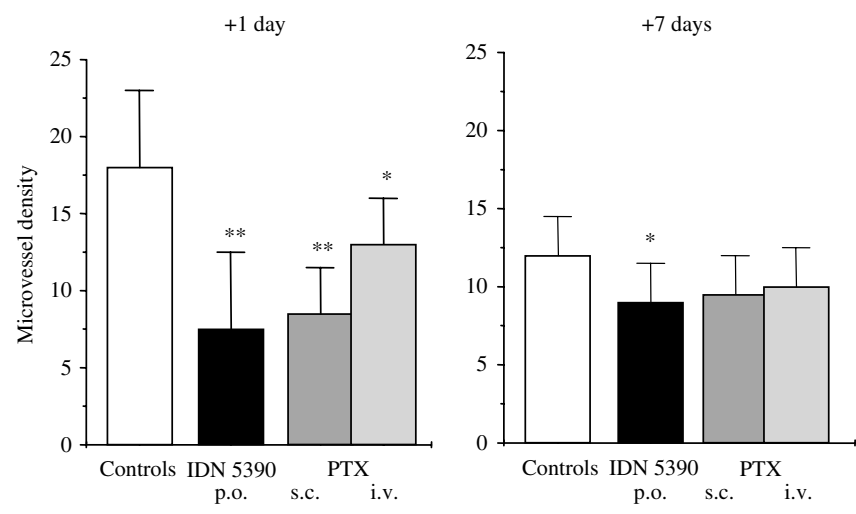

Figure 5 Microvessel density in INT.ACP/PTX tumour xenograft after IDN 5390 or PTX treatment. Tumours were implanted s.c. in mice and treated with: solvent (empty column); IDN $5390120 \mathrm{mg} \mathrm{kg}^{-1}$, per os, daily $\times 8$ (black column); PTX $10 \mathrm{mg} \mathrm{kg}^{-1}$, s.c., daily $\times 8$ (dark grey column); PTX $54 \mathrm{mg} \mathrm{kg}^{-1}$, i.v., $\mathrm{q} 4 \mathrm{~d} \times 3$ (light grey column). At I or 7 days after last treatment, tumours were surgically removed, and MVD was evaluated by immunoistochemical analysis (see Materials and Methods). Columns represent the mean $M V D \pm$ s.d. $* P<0.05$, $* * P<0.000$ I vs control tumours, by Students' t-test.

novel taxane was assessed in INT.ACP/PTX tumour xenografts (Table 2 and Figure 5). The MVD analysis performed one day after the last drug treatment $(+1)$ clearly showed that both drugs, delivered by the daily schedule, significantly decreased MVD $v s$ control tumours (55 and 50\% MVI, respectively; $P<0.0001$ for both). Paclitaxel was administered daily by the s.c. route, due to its very poor oral bioavailability (Sparreboom et al, 1997; Polizzi et al, 2000). After 1 week $(+7)$, a significant inhibitory effects on MVD was still present only in the IDN 5390 -treated tumours $(P<0.05)$. Paclitaxel administered i.v. at its best regimen for antitumour activity (high-dose/intermittent schedule) had limited effect on tumour angiogenesis.

\section{DISCUSSION}

Resistance to taxanes involves several mechanisms including mdr gene amplification or overexpression and alterations in drugtarget interactions (Horwitz et al, 1993). Indeed, mdr-1 gene expression has been associated with the development of PTX resistance in patients with ovarian cancer (Kamazawa et al, 2002). In contrast, $\beta$-tubulin mutations have been reported to be rare in newly diagnosed and in recurrent PTX-resistant human ovarian cancer patients (Lamendola et al, 2003). The tumour line investigated in the study, INT-ACP/PTX, was originated following in vivo treatment with PTX. Such a model, as far as we know, is the first experimental system where tumour resistance to PTX was acquired in vivo, using therapeutic doses of the drug. Our results indicate that in the INT.ACP/PTX tumour cells the resistance to PTX was mediated by expression of Pgp, because tumour cells displayed an increased expression of Pgp protein compared to the parental cell line A2780/DDP. The high susceptibility of A2780/ DDP cells to acquire an MDR phenotype was supported by the increased expression of Pgp observed after treatment with taxanes in such cells.

Recently, Bcl-2 downregulation has been reported as another resistance mechanism to PTX (Ferlini et al, 2003). The Bcl-2 protein is downregulated in the resistant INT.ACP/PTX cells compared to the A2780/DDP cells. However, the relevance of this finding in the resistant phenotype remains to be defined. Indeed, the almost complete reversal of resistance to PTX by verapamil supports a typical MDR-mediated resistance.

In the present study IDN $5390,120 \mathrm{mg} \mathrm{kg}^{-1}$, administered once a day for 8 days induced a marginal tumour response (60\% TWI), whereas a superior antitumour effect ( $87 \%$ TWI) was achieved by the same dose twice a day for 2 weeks (Pratesi et al, 2003). Indeed, the very low toxicity of the compound allows very frequent drug administration and very prolonged period of treatment. Indeed, total doses up to $4.5 \mathrm{~g} \mathrm{~kg}^{-1}$, that is, 20-fold higher than those of PTX, were well tolerated (Pratesi et al, 2003). Thus, in spite of the recognition by transport systems detected in cell systems (Distefano et al, 1998; our results), a contribution of cytotoxic effect in antitumour activity of IDN 5390 could be expected. Indeed, the previously reported plasma levels of IDN 5390 in mouse $\left(C_{\max }\right.$, $25 \mu \mathrm{g} \mathrm{ml}^{-1}$ ) after oral administration of a single dose $\left(120 \mathrm{mg} \mathrm{kg}^{-1}\right)$ are high enough to support our interpretation (Pratesi et al, 2003).

Tumour growth inhibition by IDN 5390 was associated with a strong reduction of tumour MVD, which was more evident 1 day after treatment end (55\% MVI) and still present 1 week after $(26 \%$ MVI). Such finding was in keeping with other preclinical in vivo studies on inhibition of tumour angiogenesis by various cytotoxic drugs delivered by the so-called 'metronomic chemotherapy', that is, frequent and prolonged administration schedule in contrast to the conventional high-dose/intermittent-schedule regimen (Hanahan et al, 2000; Schirner, 2000; Miller et al, 2001). Indeed, the conventional regimen of PTX (i.v., high-dose/intermittent-treatment) compared to the daily schedule, achieved a superior level of antitumour activity (31 vs 0\% TWI), but a lower effect on MVD. Inhibition of angiogenesis in the INT.ACP/PTX tumour xenograft was achieved also by PTX delivered by the daily s.c. treatment. The reduction on MVD was similar to that achieved by the analogue, but PTX-induced effect was less persistent. No antitumour efficacy was achieved by PTX delivered by these conditions. In spite of a similar ability to inhibit tumour angiogenesis, the limited exposure of the tumour cells to PTX, due to the low drug tolerability in vivo, may account for the lack of efficacy of PTX. In addition, the higher level of resistance (RI 15 vs 7 for PTX and IDN 5390, respectively) may be a further drawback of PTX. Moreover, considering the very poor oral bioavailability of PTX (Sparreboom et al, 1997; Polizzi 
et al, 2000), the s.c. route was used for the daily treatment, and only $10 \mathrm{mg} \mathrm{kg}^{-1}$ were tolerated, due to the high vesicant activity of the drug (Pratesi et al, 2003). In contrast, the possibility of delivering repeated administrations of IDN 5390 allows keeping tumour angiogenesis under control for long time. The effects of IDN 5390 on tumour angiogenesis in vivo are consistent with its ability to inhibit endothelial cell motility (Taraboletti et al, 2002) as well as to downregulate the expression of angiogenic growth factors (Pratesi et al, 2003). Downregulation of VEGF and bFGF expression, already reported in a glioma cell line (Pratesi et al, 2003), was observed even in INT-ACP/PTX cells (not shown). Thus, the responsiveness of the tumour to IDN 5390 may be the result of the contribution of both antiangiogenic and cytotoxic effects.

In conclusion, the relevant antitumour activity of the novel taxane IDN 5390 against a PTX-resistant tumour xenograft is likely to be the result of multiple actions, favoured by the peculiar pharmacological properties of the compound. Indeed, the good tolerability in vivo and the oral bioavailability allow exploiting the therapeutic potential of high doses of the drug (possibly allowing direct cytotoxicity) throughout a continuous and prolonged period of treatment (favouring effect on angiogenesis inhibition). Although the therapeutic advantages of IDN 5390 over PTX could be achieved only at relatively high dose levels, the favourable pharmacological profile makes IDN 5390 a promising candidate for clinical development.

\section{ACKNOWLEDGEMENTS}

This work was partially supported by the Associazione Italiana Ricerca sul Cancro, Milan, by the MIUR (FIRB Project), Rome, by the Ministero della Salute, Rome and by the T Hoepli Foundation, Italy. We thank Ms L Zanesi for editorial assistance.

\section{REFERENCES}

Beherens BC, Hamilton TC, Masuda H, Grotzinger KR, Whang-Peng J, Louie KG, Knutsen T, McKoy WM, Young RC, Ozols RF (1987) Characterization of a cis-diamminedichloroplatinum(II)-resistant human ovarian cancer cell line and its use in evaluation of platinum analogues. Cancer Res 47: 414-418

Belotti D, Vergani V, Drudis T, Borsotti P, Pitelli MR, Viale G, Giavazzi R, Taraboletti G (1996) The microtubule-affecting drug paclitaxel has antiangiogenic activity. Clin Cancer Res 2: 1843-1849

Browder T, Butterfield CE, Kralling BM, Shi B, Marshall B, O'Reilly M, Folkman J (2000) Antiangiogenic scheduling of chemotherapy improves efficacy against experimental drug-resistant cancer. Cancer Res 60: $1878-1886$

Cassinelli G, Lanzi C, Supino R, Pratesi G, Zuco V, Laccabue D, Cuccuru G, Bombardelli E, Zunino F (2002) Cellular bases of the antitumor activity of the novel taxane IDN 5109 (BAY59-8862) on hormone-refractory prostate cancer. Clin Cancer Res 8: 2647-2654

Distefano M, Scambia G, Ferlini C, Gallo R, De Vincenzo R, Filippini P, Riva A, Bombardelli E, Mancuso S (1998) Antitumor activity of paclitaxel (taxol) analogues on MDR-positive human cancer cells. Anti-Cancer Drug Design 13: 489-499

Ferlini C, Raspaglio G, Mozzetti S, Distefano M, Filippetti F, Martinelli E, Ferrandina G, Gallo D, Ranelletti FO, Scambia G (2003) Bcl-2 downregulation is a novel mechanism of paclitaxel resistance. Mol Pharmacol 64: $51-58$

Fidler IJ, Ellis LM (2000) Chemotherapeutic drugs - more really is not better. Nat Med 6: 500-502

Gottesman MM, Pastan I (1993) Biochemistry of multidrug resistance mediated by the multidrug transporter. Annu Rev Biochem 62: 385-427

Hanahan D, Bergers G, Bergsland E (2000) Less is more, regularly: metronomic dosing of cytotoxic drugs can target tumor angiogenesis in mice. J Clin Invest 105: $1045-1047$

Horwitz SB, Cohen D, Rao S, Ringel I, Shen H-J, Yang C-PH (1993) Taxol: mechanisms of action and resistance. J Natl Cancer Inst Monogr 15: $55-61$

Jordan MA, Toso RJ, Thrower D, Wilson L (1993) Mechanism of mitotic block and inhibition of cell proliferation by taxol at low concentrations. Proc Natl Acad Sci USA 90: 9552-9556

Kamazawa S, Kigawa J, Kanamori Y, Itamochi H, Sato S, Iba T, Terakawa N (2002) Multidrug resistance gene-1 is a useful predictor of paclitaxelbased chemotherapy for patients with ovarian cancer. Gynec Oncol 86: $171-176$

Lamendola DE, Duan Z, Penson RT, Oliva E, Seiden MV (2003) Beta tubulin mutations are rare in human ovarian carcinoma. Anticancer Res 23: $681-686$
Miller KD, Sweeney CJ, Sledge GW (2001) Redefining the target: chemotherapeutics as antiangiogenics. J Clin Oncol 19: 1195-1206

Polizzi D, Pratesi G, Monestiroli S, Tortoreto M, Zunino F, Bombardelli E, Riva A, Morazzoni P, Colombo T, D’Incalci M, Zucchetti M (2000) Oral efficacy and bioavailability of a novel taxane. Clin Cancer Res 6: 2070 2074

Polizzi D, Pratesi G, Tortoreto M, Supino R, Riva A, Bombardelli E, Zunino F (1999) A novel taxane with improved tolerability and therapeutic activity in a panel of human tumor xenografts. Cancer Res 59: 1036-1040

Pratesi G, Laccabue D, Lanzi C, Cassinelli G, Supino R, Zucchetti M, Frapolli R, D'Incalci M, Bombardelli E, Morazzoni P, Riva A, Zunino F (2003) IDN 5390: an oral taxane candidate for protracted treatment schedules. Br J Cancer 88: 965 -972

Pratesi G, Manzotti C, Tortoreto M, Prosperi E, Zunino F (1989) Effects of 5 -FU and cis-DDP combination on human colorectal tumor xenografts. Tumori 75: $60-65$

Rowinsky EK (1997) The development and clinical utility of the taxane class of antimicrotubule chemotherapy agents. Annu Rev Med 48: 353-374

Schirner M (2000) Antiangiogenic chemotherapeutic agents. Cancer Metast Rev 19: $67-73$

Sparreboom A, van Asperen J, Mayer U, Schinkel AH, Smit JW, Meijer DK, Borst P, Nooijen WJ, Beijnen JH, van Tellingen O (1997) Limited oral bioavailability and active epithelial excretion of paclitaxel (Taxol) caused by P-glycoprotein in the intestine. Proc Natl Acad Sci USA 94: $2031-2035$

Sweeney CJ, Miller KD, Sissons SE, Nozaki S, Heilman DK, Shen J, Sledge Jr GW (2001) The antiangiogenic property of docetaxel is synergistic with a recombinant humanized monoclonal antibody against vascular endothelial growth factor or 2-methoxyestradiol but antagonized by endothelial growth factors. Cancer Res 61: 3369-3372

Taraboletti G, Micheletti G, Rieppi M, Poli M, Turatto M, Rossi C, Borsotti P, Roccabianca P, Scanziani E, Nicoletti MI, Bombardelli E, Morazzoni P, Riva A, Giavazzi R (2002) Antiangiogenic and antitumor activity of IDN 5390, a new taxane derivative. Clin Cancer Res 8: $1182-1188$

Verweij J, Clavel M, Chevalier B (1994) Paclitaxel (Taxol) and docetaxel (Taxotere): not simply two of a kind. Ann Oncol 5: 495-505

Workman P, Twentyman P, Balkwill F, Balmain A, Chaplin D, Double J, Embleton J, Newell D, Raymond R, Stables J, Stephens T, Wallace J (1998) United Kingdom Coordinating Committee on Cancer Research (UKCCCR) guidelines for the welfare of animals in experimental neoplasia (second edition). Br J Cancer 77: 1-10

Zunino F, Cassinelli G, Polizzi D, Perego P (1999) Molecular mechanisms of resistance to taxanes and therapeutic implications. Drug Res Updates 2: $351-357$ 